\title{
The Role of Bacterial Exopolymers in Metal Sorption and Reduction
}

\author{
Alice Dohnalkova*, Matthew J. Marshall*, David W. Kennedy*, Yuri A. Gorby*, Liang Shi*, Alex \\ Beliaev*, Robert Apkarian** and Jim K. Fredrickson* \\ * Environmental Microbiology, Pacific Northwest National Laboratory, P7-50, Richland, WA 99354 \\ ** Integrated Microscopy and Microanalytical Facility, Emory University, Atlanta, GA 30322
}

The dissimilatory metal reducing bacterium, Shewanella oneidensis strain MR-1 has been extensively studied for the past decade for its significant capacity to utilize a wide range of organic compounds and metals during anaerobic respiration. The primary site of electron transport in gramnegative bacteria is usually the inner membrane and the periplasm, the region between inner and outer membranes with very high enzymatic activity [1]. Previous reports have suggested that multiheme cytochromes localized in the outer membrane by the type II protein secretion pathway (T2S) are involved in extracellular metal reduction [2]. In addition to its ability to reduce metals, MR-1 produces extracellular polymers that become heavily mineralized with nanocrystalline uraninite $\left(\mathrm{UO}_{2}\right)$ following reduction of $\mathrm{U}(\mathrm{VI})$. However, the exact ultrastructure, composition, and mechanisms of cellular and extracellular components involved in metal reduction and precipitation are not completely understood. To evaluate the role of the T2S in reduction and localization of $U$ by Shewanella MR-1 as well as several mutants within key genes associated with the T2S were investigated. Resting cell suspensions were incubated with U(VI) for $24 \mathrm{~h}$, and then processed anaerobically for TEM [3]. Morphological studies of these cultures showed differences in the sites of U(IV) deposition in the MR-1 compared to the mutants. Additionally, cells were often surrounded by thin $(\mathrm{nm})$ filamentous structures that were heavily covered with nanocrystalline uraninite [Fig. 1]. Previous studies report metal-binding properties of Pseudomona putida with indications that its polysaccharides (PS) are capable of binding divalent metal cations such $\mathrm{Cd}$ and $\mathrm{Pb}[4]$. Here we report this capacity in Shewanella, and investigate the hypothesis that extracellular polymeric substances are able to bind and reduce metals in association with specific lipoproteins that include $c$ type cytochromes.

Extracellular polymers in bacteria, such as exopolysaccharides (EPS) and lipopolysaccharides (LPS) are notoriously difficult to process for conventional electron microscopy. Traditional SEM and TEM require complete dehydration of cells prior examination, and often compromises delicate features of hydrated structures. Furthermore, the collapse of such structures during processing may introduce artifacts, leading to possible misinterpretations. The most accurate structural method for preserving bacterial extracellular polymers involves maintaining cells in frozen-hydrated state for observation by cryo-SEM [Fig 2]. In addition to the anoxic TEM processing of cells incubated for 24 hours with $\mathrm{U}(\mathrm{VI})$, a method using ruthenium red, osmium tetraoxide, lysine and alcian blue was adapted for visualization of EPS in ultra thin sections of cells without uranium [5]. A di-aminobenzidine (DAB) peroxidase stain was also used to localize heme-containing proteins, such as $c$-type cytochromes.

Our preliminary results revealed that the role of MR-1 EPS in U precipitation is more than simply providing sites for sorption of metal ions and nucleation of metal precipitates. Although electrostatic attraction of metal cations to the negatively charged regions of EPS components such as polysaccharide and LPS can be a dominant factor in metal binding, the apparently complex nature of the MR-1 EPS suggests that there are multiple factors involved including electron transfer. The 
ability of the extracellular polymers to bind and possibly reduce heavy metals and radionuclides may represent a novel feature of these organisms that has important implications for anaerobic respiration and biogeochemical cycling of metals.

\section{References}

[1] J.K. Fredrickson et al., Geochim. Cosmochim. Acta, 66: 3247-3262 (2002)

[2] D. R. Lovley et al., Appl. Environ. Microbiol., 59: 3572-3576 (1993).

[3] A. Dohnalkova et al, Microsc. Microanal. Vol 7, Suppl.2, 756-7 (2001)

[4] Chen, J.-H. et al, Water Res 29: 421-430 (1995).

[5] T.A. Fassel et al, Methods in Enzymology, Vol 310 Biofilms, Academic Press (1999)

[6] This research was funded by the Natural and Accelerated Bioremediation Research (NABIR) program sponsored by US Department of Energy. TEM imaging and analysis was conducted at the Environmental Molecular Sciences Laboratory (EMSL), a national scientific user facility sponsored by DOE at PNNL in Richland, WA.
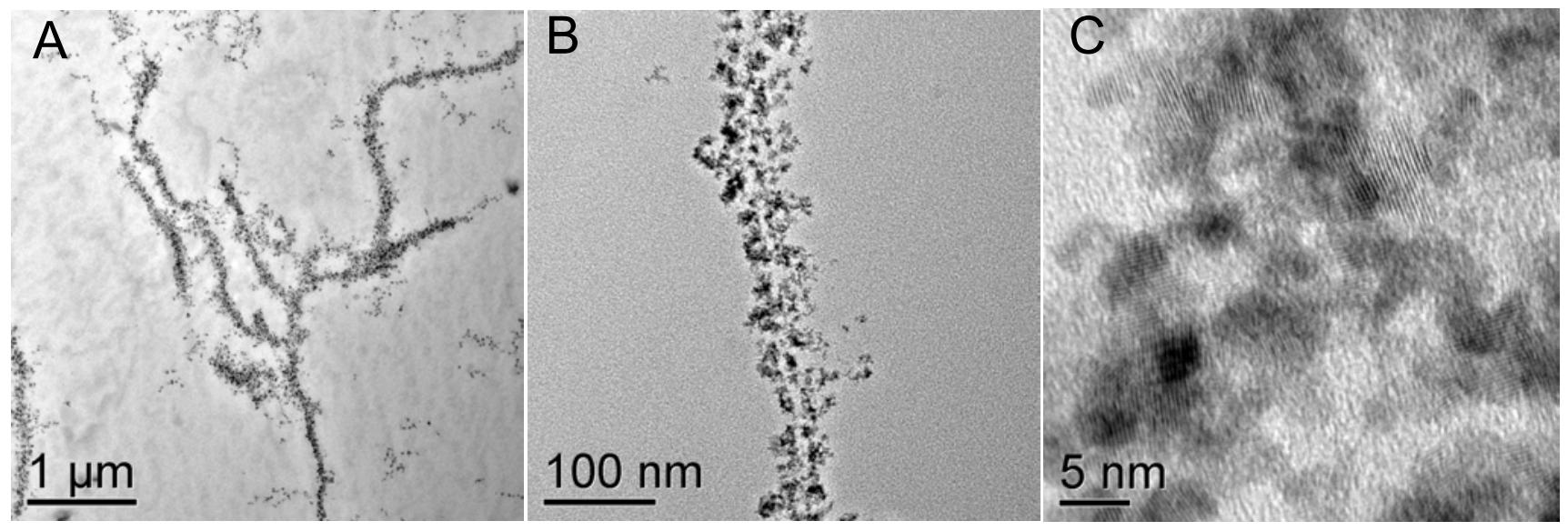

Figure 1. (A) Branching structure of extracellular polymers with heavy accumulation of $\mathrm{UO}_{2}$ particles. (B) Inset area of (A) shows that the EPS structure that is consistent with a layer of phospholipids and sugars of an unstained lipid layer (light line, center), and electron-dense nanocrystalline $\mathrm{UO}_{2}$ nanoparticles. (C) High resolution image of biogenic nanocrystalline $\mathrm{UO}_{2}$ that is very similar to particles associated with bacterial membranes.
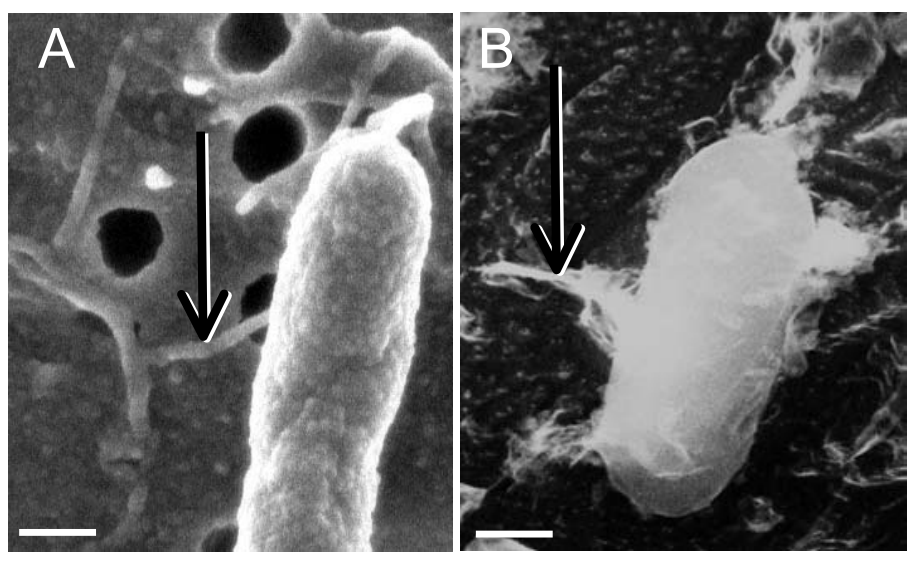

Figure 2. SEM image of a dehydrated MR-1 on filter (A), and a cell imaged in frozen-hydrated state (B). The extracellular polymers appear condensed into strands in A, a suspected artifact of dehydration. Scale bar 200 nm. 